\title{
Concerning Quality of Life Questionnaires in Breast Cancer-Related Lymphedema Patients: Review of the Literature by Cornelissen et al.
}

\author{
Tessa De Vrieze, PT, MT,1,2 Nick Gebruers, PhD, PT, ${ }^{2,3}$ and Nele Devoogdt, PhD, PT $1,4,5$
}

W ITH GREAT INTEREST we have read the recently published review by Cornelissen et al. ${ }^{1}$ In that review, a systematic literature search was performed to provide a structured overview within the plethora of quality of life $(\mathrm{QoL})$ questionnaires applied in patients with breast cancer-related lymphedema (BCRL). In view of this, the aim was to assess which questionnaire(s) is/are the most complete and accurate for this population, not only based on its clinimetric properties but also based on the amount of different aspects of QoL that are being taking into account. In our opinion, this research is of tremendous value since the use of adequate patient-reported outcome measures provides a better understanding of a patient's condition in general. The sole use of objective measurements quantifying the amount of lymphedema, on the contrary, lacks the power to encounter the real burden of lymphedema.

The conclusion of this review showed that the Lymphedema Functioning, Disability and Health questionnaire for the upper limb (Lymph-ICF), together with the Lymphedema Quality of Life Inventory (LyQLI), seems to be the two most complete and accurate questionnaires for examining QoL in patients with BCRL. Hereby was justly mentioned that "the Lymph-ICF investigates all predefined QoL domains, except the sexual functioning domain." This aspect is making no part of the questionnaire because, during the development phase of the Lymph-ICF questionnaire, none of the patients reported sexual dysfunction as a missing complaint. Nevertheless, we are absolutely conscious of the fact that, for many women, lymphedema can be an embarrassing morbidity after the treatment of breast cancer. It induces not only functional impairments but it can also entail a negative impact on psychosocial well-being with occurrence of psychosocial problems such as depression, low self-esteem, social avoidance, a decrease in self-confidence and/or an altered body image, ${ }^{2,3}$ which can be reflected in sexual concerns and issues. ${ }^{4}$ To admit the latter, a certain amount of self-confidence is needed.
Besides assessing various QoL domains, the Lymph-ICF was the only questionnaire examining all nine arm symptoms as defined in the review of Cornelissen et al. It is considered to be the only lymphedema-specific questionnaire that makes use of a visual analogue scale (VAS) score, as opposed to categorical scoring methods applied in the other mentioned lymphedema-specific questionnaires. Hereby, the difference between both answering types is being discoursed as well, as stated in the discussion section: "A VAS is a commonly used method to rate subjective experiences with the advantage of having a wide score range and high sensitivity, however, an important disadvantage is that they have a lower completion rate than other rating scales." 5

Despite the abovementioned disadvantage of having a lower completion rate, the Lymph-ICF questionnaire has proven to be a valid and reliable Dutch questionnaire in patients with BCRL. ${ }^{6}$ However, patients mentioned that the use of a scoring system with gradation like a numeric rating scale (NRS) would be an easier scoring method instead of the current VAS scoring system. Therefore, in 2014 when the Lymph-ICF-LL questionnaire for lower limbs was developed, the scoring mechanism was revised by implementing an NRS instead of a $\mathrm{VAS}^{7}$ since research also concealed that an NRS is related to a higher compliance, better responsiveness with lower error rate, and better applicability than VAS. ${ }^{8}$

For that reason, and as a reply to the valid remark of Cornelissen et al. regarding the answering type of the original Lymph-ICF, we would like to inform the authors that recently, revision of the scoring mechanism of the Lymph-ICF has been extended, resulting in the Lymph-ICF-UL questionnaire that contains an NRS instead of a VAS. Research has shown that this revised questionnaire entails an improved way of scoring, thereby facilitating completion of the questionnaire. Moreover, it has also shown very good (reliability) to good (validity) clinimetric properties in patients with BCRL. We would like to

\footnotetext{
${ }^{1}$ Department of Rehabilitation Sciences, KU Leuven-University of Leuven, Leuven, Belgium.

${ }_{2}^{2}$ Department of Rehabilitation Sciences and Physiotherapy, MOVANT, University of Antwerp, Antwerp, Belgium.

${ }^{3}$ Multidisciplinary Oedema Clinic, University of Antwerp and Antwerp University Hospital, Antwerp, Belgium.

${ }^{4}$ Lymphovenous Centre, Department of Vascular Surgery, UZ Leuven-University Hospitals Leuven, Leuven, Belgium.

${ }^{5}$ Lymphovenous Centre, Department of Physical Medicine and Rehabilitation, UZ Leuven-University Hospitals Leuven, Leuven, Belgium.
} 
thank the authors for their elucidating review as a contribution of great relevance to the literature of this important topic.

\section{Author Disclosure Statement}

No competing financial interests exist.

\section{References}

1. Cornelissen AJM, Kool M, Keuter XHA, et al. Quality of life questionnaires in breast cancer-related lymphedema patients: Review of the literature. Lymphat Res Biol 2018; 16: 134-139.

2. Pusic AL, Cemal Y, Albornoz C, et al. Quality of life among breast cancer patients with lymphedema: A systematic review of patient-reported outcome instruments and outcomes. J Cancer Surviv 2013; 7:83-92.

3. Morgan PA, Franks PJ, Moffatt CJ. Health-related quality of life with lymphoedema: A review of the literature. Int Wound J 2005; 2:47-62.

4. Winch CJ, Sherman KA, Koelmeyer LA, Smith KM, Mackie H, Boyages J. Sexual concerns of women diagnosed with breast cancer-related lymphedema. Support Care Cancer 2015; 23: 3481-3491.

5. Hauser K, Walsh D. Visual analogue scales and assessment of quality of life in cancer. J Support Oncol 2008; 6:277-282.
6. Devoogdt N, Van Kampen M, Geraerts I, Coremans T, Christiaens MR. Lymphoedema functioning, disability and health questionnaire (Lymph-ICF): Reliability and validity. Phys Ther 2011:944-957.

7. Devoogdt N, De Groef A, Hendrickx A, et al. Lymphoedema functioning, disability and health questionnaire for lower limb lymphoedema (Lymph-ICF-LL): Reliability and validity. Phys Ther 2014; 94:705-721.

8. Hjermstad MJ, Fayers PM, Haugen DF, et al. Studies comparing numerical rating scales, verbal rating scales, and visual analogue scales for assessment of pain intensity in adults: A systematic literature review. J Pain Symptom Manage 2011; 41:1073-1093.

Address correspondence to: Tessa De Vrieze, PT, MT Department of Rehabilitation Sciences Catholic University of Leuven O\&N IV Herestraat 49, Box 1510 Leuven 3000 Belgium

E-mail: tessa.devrieze@kuleuven.be 